\title{
Tissue Expression of Neuregulin-1 in Hypertrophic Scars and Keloids before and after Intralesional Bleomycin Injection
}

\author{
N.E.Sorour ${ }^{1}$, N.F.El Husseini ${ }^{2}$, A.I.Mustafa ${ }^{1}$ and H.M.Abdullah ${ }^{1}$ \\ ${ }^{1}$ Dermatology and Andrology Dept., Faculty of Medicine, Benha Univ., Benha, Egypt \\ ${ }^{2}$ Medical Biochemistry and Molecular Biology Dept., Faculty of Medicine, Benha Univ., Benha, Egypt \\ E-Mail: H.M6@gmail.com
}

\begin{abstract}
Hypertrophic scars and keloids are forms of abnormal wound healing. Both entities are the result of exuberant fibroblast response in the dermis. Nevertheless, their clinical and histopathological characteristics, as well as their pathogenesis, are differentThe aim of this study was to study the role of neuregulin- 1 in the pathogenesis of hypertrophic scars and keloids and studying the change in tissue expression after intralesional bleomycin injection. This prospective case - control study was carried on20patients who were suffering from keloids or hypertrophic scarsand were treated by intra lesional bleomycin injection, in addition to 20 apparently healthy individuals as a control group. Assement of hypertrophic scars and keloids before and after treatment byVancouver scar scale laboratory investigations including punch biopsies from hypertrophic scars, keloids and normal tissues, qRT-PCRfor gene expression of Neuregulin1 RNA was done. HTS group before treatment showed significantly higher level of neuregulin 1 but significantly lower after treatment and neuregulin 1 sensitivity was $80 \%$, specificity was $100 \%$,and accuracy was 90\%.Neuregulin 1 before treatment showed significant positive correlation with Neuregulin 1after treatment.Better response was significantly associated with Lower baseline neuregulin 1.Higher neuregulin 1 was considered as risk predictor of HTS or keloid susceptibility and severity. Bleomycin usage has a better result and minimal complications and less recurrence rate, which shows its usefulness as the first-line treatment modality for management of keloids and hypertrophic scars.
\end{abstract}

\section{Introduction}

Keloids and hypertrophic scars are an overactive condition of the skin tissue to early lesions characterized by proliferation of fibroblasts and excessive production of collagen in the lesion [1]. They caused by cutaneous injury and irritation, including trauma, acne, insect bite, surgery, burn, vaccination, skin piercing, chicken pox, and herpes zoster infection [2].

Hypertrophic scars are typically raised, red or pink, and sometimes pruritic but do not exceed the margins of the original wound.Keloids can have side effects such as interfering with movement, pain, and itching. They may be more common in people with darker skin tones and do not resolve or fade with time [3].

Although the pathogenesis of excessive wound healing is not fully elucidated, clinical experience suggests that excessive wound healing is an aberrant form of wound healing, which may occur as a result of dysregulation in one of the three phases of wound healing, and is characterized by continuously localized inflammation [4]. Excessive wound healing often involves an exaggerated function of fibroblasts and excess accumulation of ECM during wound healing [5].

Neuregulins (NRGs) are a family of four structurally related proteins that are part of the epidermal growth factors ( EGF) family of proteins. These proteins have been shown to have diverse functions in the development of the nervous system and play multiple essential roles in vertebrate embryogenesis including: cardiac development, Schwann celland oligodendrocyte differentiation, some aspects of neuronal development, as well as the formation of neuromuscularsynapses. They can directly bind to only the ErbB3 and ErbB4 receptors, which can then heterodimerize with ErbB2 (an orphan receptor) and ErbB1 to activate and/ or potentiate subsequent signaling activity [6].

It is known that NRGs regulate proliferation ,differentiation, a migration keratinocytes via their receptors . Neuregulin 1 (NR G1), secreted from fibroblasts and plays a role in skin pigmentation, keratinocyte migration in wound healing, hypertrophic scarring and fibrosis. It was suggested that NRG1 is highly expressed in fibroblasts derived from darker skin and regulates the alteration of skin pigmentation [7].

Bleomycin is a cytotoxic antibiotic derived from Streptomyces verticellus. Ithas antineoplastic,antibacterial and antiviral propertiesand has been used for many years in dermatologicalpractice for treating recalcitrant plantar warts, cutaneous neurofibromas and keratoacanthomas.In addition, it is frequentlyused for treating various malignancies. Morerecently, its use has been focused in the treatmentof keloid and hypertrophic scars [8].

Bleomycin is thought to reduce collagen synthesis, increase degradation secondary to the inhibition of lysyl oxidase, a cross-linking enzyme involved in the maturation of collagen and TGF- $\beta 1$, and to induce fibroblast apoptosis. The main mode of action through which bleomycin blocks the cell cycle is via the inhibition of DNA synthesis, RNA, and protein synthesis as well as the production of reactive oxygen species [9].

The aim of this study was to study the role of neuregulin- 1 in the pathogenesis of hypertrophic scars and keloids and studying the change in tissue expression after intralesional bleomycin injection. 


\section{Patient and method}

This study was prospective case-control study included 20patients who were suffering from keloids or hypertrophic scars and were treated by intra lesionalbleomycin injection. In addition, 20 apparently healthy individuals of matched age and sex as a control group. patients were selected from the Outpatient Clinic of Dermatology and Andrology Department of Benha University Hospital,.from(March2019 to March 2020)..

All patients were enrolled in the study must have clinically confirmed keloids and hypertrophic scars with:

1 -Age $\geq 18$ years old.

2-Acceptable pretreatment laboratory studies as complete Blood cells count (CBC),liver function, renal function and blood sugar.

\section{Any subject was excluded from the study if he/she} was

- Pregnant woman or if want to be pregnant.

- Lactating woman.

- Suffering from hepatic dysfunction and renal dysfunction.

- Having active infectious disease and complaining from blood dyscrasias.

\section{All participants were divided into two groups}

- Group I:20 patients suffering from hypertrophic scarsand keloids who treated by intralesionalbleomycininjection .

- Group II:20 apparently healthy individuals of matched age and sex as a control group .

\section{All patients were subjected to full history taking and dermatological examination}

Treatment protocol intralesional bleomycin was included 20 twenty patients were injected intralesionally with bleomycin at a concentration of 1.5 $\mathrm{IU} / \mathrm{ml}$. Initially, local anesthetic (MepivacaineHCl 3\%) was administered at the lesion site. Then, multiple intralesional injections of bleomycin at a dose of $0.5-1$ $\mathrm{ml} / \mathrm{cm} 2$ with a maximum dose of $4 \mathrm{ml}$ per session using insulin syringe was administered with two week interval for 4 consecutive sessions.

All patients were followed up monthly for six months after the last sessions to be inspected for any recurrence, complication and side effects, as pain at the site of injection,hyperpigmentation,ulceration, and infection

Tissue sampling : Hypertrophic scars,keloids, and normal tissue were carefully excised by $4-\mathrm{mm}$ punch biopsies then immediately put in liquid nitrogen sample and stored at- $80^{\circ} \mathrm{c}$ for further assessment of NRG1 gene

Genotyping : Molecular biology investigation: Relative mRNA expression levels of neuregulin1 gene measured quantitatively in hypertrophic scars and keloid from patients and controls. Also, assessment of tissue expression of neuregulin1 was done after 4 sessions of treatment with intralesional bleomycin injection.

\section{Results}

The present study included 20 HTSs and keloids patients and 20 healthy control subjects.

The mean age of HTS group was 27.3 years, they were 11 males (55\%) and 9 females (45\%). In addition to 20 healthy control group, their mean age was 26.9 years, they were 9 males (45\%) and 11 females (55\%). Patients and control groups had matched age and gender (p>0.05 for each).

Many precipitating factors in patients with HTS and keloids were noticed,7( 35\%) had wound,5( 25\%) had surgery, $4(20 \%)$ had burn, $3(15 \%)$ were smokers, 1( 5\%) had insect bite and 3(15\%) were spontaneous .

Mean disease duration was 1.9 years. Many site were affected, head, face in $4(20 \%)$, trunk in9( $45 \%)$, upper limb in 5( 25\%), lower limb in $2(10 \%$.) Movement was restricted in10( 50\%), 14(70\%) suffered of pain, 11(55\%) suffered of itching;18( 90\%) had single lesion, $1(5 \%)$ had 2 lesions, $1(5 \%)$ had 3 lesions. Mean size was $4 \mathrm{~cm} ; 2(10 \%)$ were recurrent, $5(25 \%)$ received previous treatment, out of them 1 (20\%) had laser,3 (60\%) had sacro and 1(20\%) had steroids.

Adverse effects after treatment were observed in only 4 patients (20\%) in the form of hyperpigmentation. No pain at the site of injection or ulcerations were noticed.

Pigmentation,Vascularity, pliability, height, and total score decreased significantly after treatment $(\mathrm{p}<0.001, \mathrm{p}<0.001, \mathrm{p}<0.001, \mathrm{p}<0.001$ respectively).

Improvement was calculated, mean percentage improvement in, vascularity, pliability, height, and total score was, (38.9\%),(45.8\%),( 46.7\%), and (35\%) respectively .but pigmentation did not change.,

Hypertrophic scars(HTS)and keloids group before treatment showed significantly higher level of neuregulin 1 when compared healthy control levels $(\mathrm{p}<0.001)$.

Hypertrophic scars (HTS) and keloids group after treatment showed significantly lower level of neuregulin 1 when compared before treatment levels $(\mathrm{p}<0.001)$.

No significant difference was found regarding neuregulin 1 level between HTS and keloids after treatment and healthy control groups ( $p>0.05$ ),

Receiver operating characteristic (ROC) curve of neuregulin 1 tissue expression level was conducted for discrimination between patients ( HTS and keloids) and control groups.

Neuregulin 1 showed good AUC (AUC $=0.870)$. At best cut off value of $111204.5\left(\log _{10}=5\right)$, sensitivity was $80 \%$, specificity was $100 \%$, PPV was $100 \%$, NPV was $83.3 \%$, and accuracy was $90 \%$.

No significant associations were found regarding neuregulin 1 level according to baseline data in patients( HTSand keloids) group ( $p>0.05$ for each).

Baseline neuregulin 1 level showed significant positive correlation with baseline total score $(\mathrm{p}<0.001)$. 
Neuregulin 1 level after treatment level showed significant positive correlation with total score after treatment ( $\mathrm{p}=0.003)$. as shown in figure(15-17).

Neuregulin 1 before treatment showed significant positive correlation with Neuregulin 1after treatment $(\mathrm{p}<0.001)$

Otherwise, no significant correlations of neuregulin 1 were found with other parameters in HTS and keloids group.

Logistic regression analysis was conducted for prediction of HTS development using age, gender, and baseline neuregulin 1 level as confounders. Higher neuregulin 1 was considered as risk predictor of HTS or keloid susceptibility.
Linear regression analysis was conducted for prediction of HTS or keloid severity (higher VSS score) using age, gender, duration, recurrence, previous treatment and baseline neuregulin 1 level as confounders. Higher baseline neuregulin 1 level was suggested to be independent risk predictor for HTS or keloid severity $(\mathrm{B}=2.036, \mathrm{p}=0.021)$.

Linear regression analysis was conducted for prediction of factors affecting response of treatment, using age, gender, duration, recurrence, previous treatment, baseline neuregulin 1 level as confounders. Lower baseline neuregulin 1 level was suggested to be independent predictors for better response.

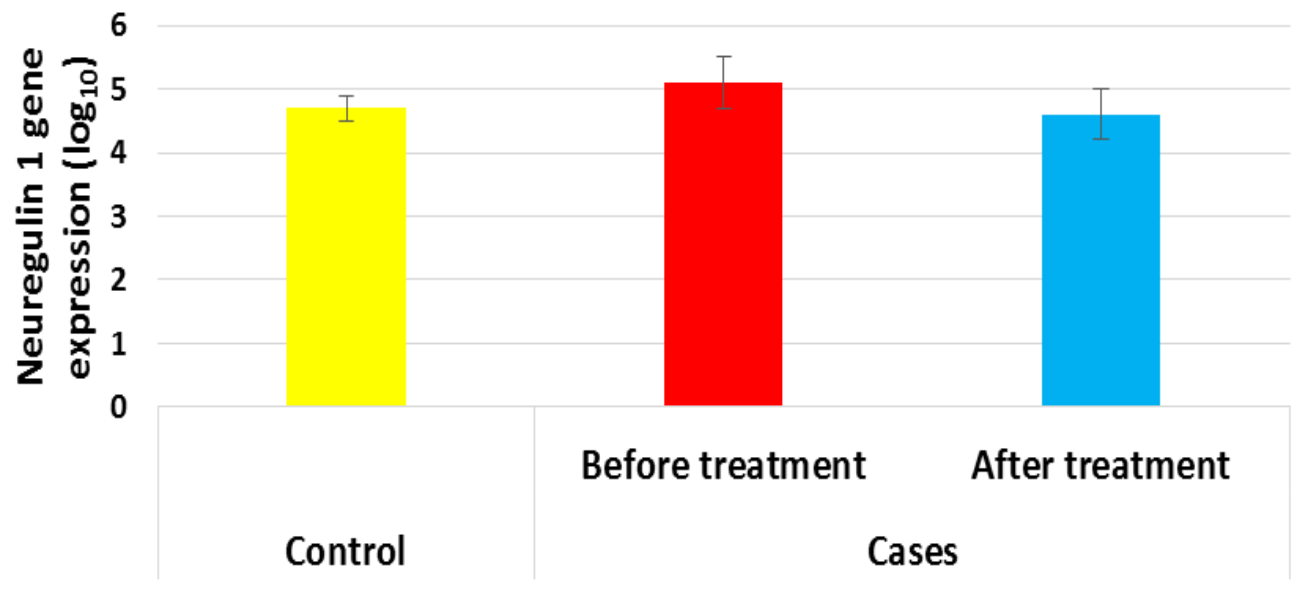

Fig (1) Bar chart for neuregulin1 tissue expression level in control as well as patients groups, before and after treatment. Bar represents mean, while error bar represents standard deviation.

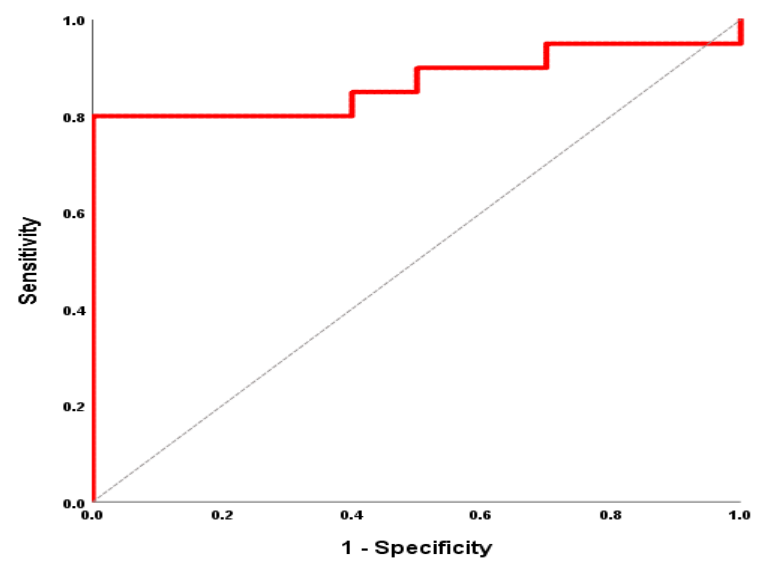

Fig (2) ROC curve of neuregulin 1 tissue expression level for discrimination between patients and control groups.

\section{Discussion}

In dermatology, intralesional bleomycin is used on an off-label basis in multiple skin conditions, including keloids, hypertrophic scars, warts, hemangiomas, vascular and lymphatic malformations, telangiectasia, skin cancer and condyloma. It induces DNA damage, cell apoptosis and inhibits the synthesis of collagen due to a decrease in TGF- $\beta$ [10].

The aim of this study was to study the role of neuregulin- 1 in the pathogenesis of hypertrophic scars and keloids and studying the change in tissue expression after intralesional bleomycin injection. 
In the current study, there were improvement in vascularity, pliability, height, and total score.

Huu et al., [11] study included 55 patients having 120 keloids of different sizes and locations. The average number of injections was $3.9 \pm 1.1$. After treatment, $80.8 \%$ of patients got itchiness relief, and $73.3 \%$ of patients got pain relief. Blood vessel status, scar stiffness, and scar thickness improved at $70.6 \%$, $89.3 \%$ and $87 \%$, respectively, by the VSS scale. In particular: $70.8 \%$ of scars became completely flat, $8.3 \%$ fairly flat, $17.5 \%$ comparatively flat, $3.3 \%$ averagely flat and no poorly flat scars and this was in agreement with the current study results .

Reddy et al. [12], (study included twenty patients, treated with intralesional injection of bleomycin, 13 $(65 \%)$ showed excellent response, $3(15 \%)$ showed good response, $2(10 \%)$ showed fair response and 2 (10\%) showed poor response. There was complete resolution of symptoms in 11 patients $(55 \%)$ and improvement in the other $9(45 \%)$ and this was in accordance to the current study results .

In the current study the Vancouver scar scale showed $\pm 9.7(55 \%)$ improvement after treatment with bleomycin by where its mean was $9.1 \pm 1.4$ before treatment and $4.6 \pm 1.1$ after treatment and this was in accordance toKabel et al., [13] study where the mean Vancouver scar scale in all patients before treatment was $9.32 \pm 1.46$ and it was $2.7 \pm 0.95$ after treatment with a mean total improvement of $73 \%$.

In the current studyBaseline neuregulin 1 level showed significant positive correlation with baseline total score $(\mathrm{p}<0.001)$. Neuregulin 1 level after treatment level showed significant positive correlation with total score after treatment $(\mathrm{p}=0.003)$.

In the current study, patients group before treatment showed significantly higher level of neuregulin 1 but significantly lower after treatment and neuregulin 1 sensitivity was $80 \%$, specificity was $100 \%$, and accuracy was $90 \%$ and this was in agreement Kim et al., [7] study illustrated that NRG1 expression levels were significantly higher in hypertrophic scar(HTS) than normal skin .

Kim et al., [7] illustrated that NRG1 have a role in migration of keratinocytes during wound healing process. NRG1 exhibited higher expression in hypertrohic scar fibroblast (HTSF )than in normal fibroblast( NF cells). In addition, HER2 and HER3, receptors for NRG1, were expressed in HTSF but were not detected in NF cells. These findings indicate that cellular alteration of HTSF cells exhibit expression of NRG1 and its receptors, and hence, lead to the stimulation of autocrine signalling, it has been reported that NRG is expressed and secreted by fibroblasts derived from dark skin .

Jumper et al.; [14] demonstrated that keloid fibroblast reflect in situ KMD tissue, with increased NRG1 and ErbB2. ErbB2overexpression leads to homodimerisation and constitutive activity through autophosphorylation, leading to ligand-independent signaling .
Choi et al. [15] studied MelanoDerm skin modeland in cultured human melanocytes. They found that NRG1 increases the proliferation of human melanocytes, possibly via the phosphorylation of Akt, and increases pigmentation levels. NRG-1 is much more efficient in increasing melanin content as the melanocytes became confluent. Therefore, it seems that the initial effect of NRG-1 is to increase proliferation, and then to increase pigmentation when prolife ration becomes limiting

In the current study, higher baseline neuregulin 1 level was suggested to be independent risk predictor for HTS or keloid severity and lower baseline neuregulin 1 level was suggested to be independent predictors for better response but no previous study detected this findings.

In Kabel et al., [13] study, no correlation between clinical response and age, sex or duration of keloids was found.. On the contrary, Kontochristopoulos et al. [16] found a correlation between duration of keloids and recurrence. He observed that the response was low in older lesions.

In Kabel et al., [13] study, there was no recurrence during the follow up period.

bleomycin usage has a better or comparable result as with other treatment modalities such as Triamcinolone and silicon gel sheets, and has minimal complications and less recurrence rate, which shows its usefulness as the first-line treatment modality for management of keloids and hypertrophic scars [12].

Regarding the side effects, in Kabel et al., [13] study, hyperpigmentation was present in 42 patients (70\%), ulceration was present in 14 patients $(21.33 \%)$ and pain at the injection site was present in all patients $(100 \%)$.the current study show nouleration,pain at the site of injection tolerable pain, the difference may be due to performing injection without local anathesia.

\section{References}

[1] L.M.Kasyanju Carrero, W.W.Ma, H.F.Liu. Botulinum toxin type A for the treatment and prevention of hypertrophic scars and keloids: updated review. J. Cosmet. Dermatol, Vol.18(1), PP.10-15,2019.

[2] H.Lee, Y.Jang. Recent Understandings of Biology, Prophylaxis and Treatment Strategies for Hypertrophic Scars and Keloids. Int.J .Mol.Sci,VOL. 19(3),PP.711,2018.

[3] L.Carswell, J.Borger. Hypertrophic Scarring Keloids. In StatPearls [Internet]. StatPearls Publishing,2019.

[4] B.Berman, A.Maderal, B.Raphael. Keloids and hypertrophic scars: pathophysiology, classification, and treatment.Dermatol. Surg, Vol. 43,PP.S3-S18. ,2017.

[5] K.Ashcroft, F.Syed, A.Bayat. Site-Specific Keloid Fibroblasts Alter the Behaviour of Normal Skin and Normal Scar fibroblasts through paracrine signalling.Plos. One, Vol.8(12),PP.75600,2013. 
[6] V.Veikkolainen, K.Vaparanta, K.Halkilahti. Function of ERBB4 is determined by alternative splicing.Cell cycle,vol. 10(16), PP.26472657,2011.

[7] J.S.Kim, I.G.Choi, B.C.Lee. Neuregulin induces CTGF expression in hypertrophic scarring fibroblasts. Mol.Cell. biochem, Vol.365(1-2), PP.181-189,2012.

[8] L.Bik, T.Sangers, K.Greveling. Efficacy and tolerability of intralesional bleomycin in dermatology: A systematic review.J. Am. Acad. Dermatol,2020.

[9] W.Xi-Qiao, L.Ying-Kai, Q.Chun. A review of the effectiveness of antimitotic drug injections for hypertrophic scars and keloids. Ann. plast .surg, Vol. 63(6),PP.688-692,2009.

[10] I.Bodokh, P.Brun. Treatment of keloid with intralesional bleomycin. InAnn.Dermatol. venereal, Vol.123,( 12), PP.791-794) ,1996.

[11] N.D.Huu, S.N.Huu. X.Le . Successful treatment of intralesional Bleomycin in keloids of Vietnamese population.Open Access Maced. J. Med. Sci, Vol.7(2),PP.298,2019.

[12] R.Reddy, S.Harinatha, N.Raghunath, The role of bleomycin in management of hypertrophic scars and keloids-a clinical trial. Our. Dermatol. Online, Vol. 6(4),PP.404. ,2015.

[13] A.M.Kabel, H.H.Sabry, N.E.Sorour, F.M.Moharm. Comparative study between intralesional injection of bleomycin and 5fluorouracil in the treatment of keloids and hypertrophic scars.J. Dermatol and Dermatol. Surg, Vol. 20(1), PP.32-38. ,2016.

[14] Umper, N.; Hodgkinson, T.; Paus, R. and Bayat, A. (2017): A role for neuregulin-1 in promoting keloid fibroblast migration via ErbB2- mediated signaling Acta. Dermato-venereologica.; 97(6-7): 675-684.

[15] Choi, R.Wolber, W.Gerwat, Mann. The fibroblast-derived paracrine factor neuregulin-1 has a novel role in regulating the constitutive color and melanocyte function in human skin $\mathrm{J}$. Cell. Sci, Vol.123(18), PP.3102-3111,2010.

[16] G. Kontochristopoulos, C.Stefanaki, A. Panagiotopoulos. Intralesional 5-fluorouracil in the treatment of keloids: an open clinical and histopathologic study.J. Am. Acad. Dermatol, Vol. 52, PP.474-479,2005. 